\title{
Uterine Artery Embolization with Small-Sized Particles for the Treatment of Symptomatic Adenomyosis: A 42-Month Clinical Follow-Up
}

\author{
Kai Yuan ${ }^{1,2}$ \\ Jin Long Zhang ${ }^{2}$ \\ Jie $\mathrm{Yu} \mathrm{Yan^{2 }}$ \\ Bing Yuan' \\ Jin $\mathrm{Xin} \mathrm{Fu}^{2}$ \\ Yan Wang ${ }^{2}$ \\ Xue Dong Sun ${ }^{3}$ \\ Yang Guan ${ }^{2}$ \\ Feng Duan ${ }^{2}$ \\ Mao Qiang Wang ${ }^{1,2}$ \\ 'Medical School of Chinese PLA, Beijing, \\ I00853, People's Republic of China; \\ ${ }^{2}$ Department of Interventional Radiology, \\ The Fifth Medical Center, Chinese PLA \\ General Hospital, Beijing, I00853, \\ People's Republic of China; ${ }^{3}$ School of \\ Medicine, Nankai University, Tianjin, \\ 30007I, People's Republic of China
}

Correspondence: Mao Qiang Wang

Department of Interventional Radiology,

The Fifth Medical Center, Chinese PLA

General Hospital, 28 Fu-Xing Road,

Beijing, 100853, People's Republic of

China

Tel +86 10-66937/90

Email wangmq@vip.sina.com
Purpose: To assess the long-term outcome of performing uterine artery embolization (UAE) using small particles in women with symptomatic adenomyosis (AD).

Methods: Twenty-seven consecutive women (median age 42 years, range 29-53 years) with $\mathrm{AD}$, in eight cases $\mathrm{AD}$ combined with fibroids, who underwent UAE between February 2015 and January 2019, were retrospectively analyzed. The embolization was performed using small-sized polyvinyl alcohol particles $(100 \mu \mathrm{m}$ and $300 \mu \mathrm{m})$. The patients completed the Uterine Fibroid Symptom and Quality of Life questionnaire at baseline and at a 42-month follow-up (range 24-71). The junction zone (JZ) thickness and uterine volume were also calculated at baseline and at a three-month follow-up.

Results: The total symptom severity score (SSS) decreased from a median of 59 (range 34 78) at baseline to a median of 9 (range 3-47) at the end of this study; the health-related quality of life score (HRQOL) increased from a median of 38 (range 23-49) at baseline to a median of 84 (range 46-97) at 42 months. Twenty of the 27 patients were asymptomatic. The clinical response of the remaining seven women was little improvement in their symptoms, and one of the seven women underwent a hysterectomy at 35 months. Twentysix of the 27 (96\%) patients had a preserved uterus at the 42-month follow-up. There was no difference after UAE in SSS, HRQOL, junction zone (JZ) thickness, and uterus volume between patients with pure $\mathrm{AD}$ and those with $\mathrm{AD}$ combined with fibroids ( $p=0.729,0.710$, 0.973 , and $>0.99$ ). There was no difference in the JZ thickness and uterus volume at baseline between the asymptomatic women and the women with an insufficient response ( $p=0.854$ and 0.253), and there were no major complications afterwards.

Conclusion: From the long-term follow-ups, it could be seen that UAE using small particles is safe and effective in treating $\mathrm{AD}$, especially in preserving the uterus. There is no relationship between the clinical outcomes and the initial presence of AD, with or without fibroids, and the JZ thickness at baseline does not seem to be a predictor for the long-term outcome of UAE.

Keywords: adenomyosis, uterine artery embolization, UFS-QoL, interventional radiology

\section{Introduction}

Adenomyosis (AD) is a unique pathophysiological condition that sees the formation of ectopic endometrial glands and stroma within the myometrium. It is common in women of reproductive age, and typical symptoms include menorrhagia, chronic pelvic pain, and dysmenorrhea. ${ }^{1-3}$ Current treatment options for symptomatic AD include hysterectomy, medication, conservative surgery, and minimally invasive 
techniques, including uterine artery embolization (UAE). However, to date, hysterectomy remains the definitive treatment. $^{4}$

In recent years, several minimally invasive treatment options for $\mathrm{AD}$ have been introduced, and UAE has gained ground as an option for $\mathrm{AD}$, and it is now the most likely alternative to hysterectomy, especially in women with diffuse $\mathrm{AD} .{ }^{5,6}$ At present, there are a variety of embolization materials selected for UAE in the treatment of $\mathrm{AD}$. The embolization materials include polyvinyl alcohol (PVA) particles, tris-acryl gelatin microspheres (TGM), sodium alginate microspheres (KMG), and gelatin sponge/foam particles (GFP), and the primary sizes used are 355-500 $\mu \mathrm{m}, \quad 500-700 \mu \mathrm{m}$, and $700-900 \mu \mathrm{m} .{ }^{7}$ However, it is not clear which particular embolization material can result in better clinical outcomes.

This study analyzed the long-term clinical results of 27 women with $\mathrm{AD}$, eight of whom also had fibroids, who were embolized with small-sized PVA particles.

\section{Materials and Methods}

\section{Patients}

The Institutional Review Board of the Chinese People's Liberation Army General Hospital, Beijing, China approved this retrospective study, which was conducted in accordance with the Declaration of Helsinki. Between February 2015 and January 2019, 27 women with a median age of 42 years (range 29-53 years) were evaluated, after presenting with pelvic pain, heavy menstrual bleeding, or bulk-related symptoms, or a combination of them. The clinical diagnosis was confirmed with transvaginal ultrasound or magnetic resonance imaging. All the patients were asked to complete the standardized symptom severity and health-related quality of life questionnaire (The Uterine Fibroid Symptom and Quality of Life [UFSQoL] questionnaire) and rate the severity of their pelvic pain on the Numerical Rating Scale (NRS) before and after the UAE. Of the 27 women, 19 had pure $\mathrm{AD}$, and eight had $\mathrm{AD}$ combined with fibroids, while 25 had diffuse $\mathrm{AD}$, and two had focal $\mathrm{AD}$.

The inclusion criteria for UAE were women with adenomyosis, with or without fibroids, suffering from heavy menstrual bleeding, dysmenorrhea, and/or cycleindependent pain and bulk-related symptoms. These women had had or were receiving medical treatment which did not appear to be working, or they had decided that such treatment was not an option. ${ }^{4}$
The exclusion criteria for UAE were the suspicion or presence of a malignancy or pelvic infection, an on-going pregnancy or a desire to conceive in the future, absolute contraindication for angiography, deep infiltrating endometriosis requiring surgery or indicating a risk of intestinal stenosis, concurrent hysteroscopic removable submucous fibroids, and being under 18 years of age. ${ }^{4}$

\section{UAE Embolization}

All the patients signed informed consent before the embolization. Patient consent was obtained to review their medical records. The procedures were performed under local anesthesia using the right femoral artery puncture approach. Selective digital subtraction angiography was performed with a $4 \mathrm{~F}$ Simmons I catheter (Cordis, East Bridgewater, NJ, USA) to evaluate the hypogastric and uterine arteries. Coaxial 2.7-Fr catheters (Progreat, Terumo Corp., Tokyo, Japan) were advanced distally into the uterine artery. Selective uterine artery angiography was performed before the embolization procedure in neutral (6-8 mL contrast medium at $1.5-2 \mathrm{~mL} / \mathrm{s}$ ) to ensure that the tip of the microcatheter was inside or at the ostium of the uterine arteries.

Embolization was performed through the microcatheter using non-spherical PVA particles. PVA particles (Cook Incorporated, Bloomington, IN, USA) (1 mL) were diluted in a $30 \mathrm{~mL}$ solution of a non-ionic contrast medium (Visipaque iodixanol 320mg I/mL, GE Healthcare). In all patients, $100 \mu \mathrm{m}$ particles were used initially, followed by $300 \mu \mathrm{m}$ particles. The endpoint of the embolization was complete stasis of the blood flow in the uterine artery. After the UAE, angiography of the internal iliac artery was performed to check if other blood vessels were supplying the uterine artery, using the $4 \mathrm{~F}$ catheter. Embolization was then performed on the contralateral side using the same technique. Ovarian artery angiography was required in patients with a hypoplastic or aplastic uterine artery functionally replaced by an ovarian artery. However, only patients with an ovarian artery entering the myometrium, seen in aortography after the UAE, could be considered for ovarian artery embolization (OAE).

\section{Post-Procedural Management}

All the patients stayed in the hospital for 5-7 days for observation. During and after the UAE procedure, intravenous narcotics were administered using a patientcontrolled analgesia pump, as well as antiemetic and nonsteroidal anti-inflammatory drugs, for pain control and to 
reduce symptoms such as nausea and vomiting. Appropriate hydration was given for three days after the UAE, and antibiotics were given for one week to prevent infection.

\section{MRI at Baseline and Follow-Up}

MRI was performed before the embolization and at the three-month follow-up. The reduction of the JZ thickness and uterine volume were measured. Uterine volume was obtained by measuring length, width, and height and calculating the volume with the prolapse ellipse equation of vol $=($ length $\times$ width $\times$ height $) \times 0.52$.

\section{Technical Assessment}

Technical success was defined as the achievement of a unilateral or bilateral uterine arterial catheterization and a successful embolization.

\section{Clinical Assessment}

AD-related symptoms were evaluated in all the women before and after the UAE using the Uterine Fibroid Symptom and Quality of Life (UFS-QoL) questionnaire, which produces a symptom severity score (SSS) and a health-related quality of life (HRQOL) score. It consists of an eight-item symptom severity scale and 29 HRQOL items. The eight items of the symptom severity scale are heavy bleeding, passing blood clots, fluctuation in the duration of the menstrual period, fluctuation in the length of the monthly cycle compared to previous cycles, a feeling of tightness or pressure in the pelvic area, frequent urination during the daytime hours, frequent nighttime urination, and feeling fatigued. The 29 HRQOL items comprise six domains: concern, activities, energy/ mood, control, self-consciousness, and sexual function. Items are scored on a 5-point Likert scale, ranging from "not at all" to "a very great deal" for symptom severity items and "none of the time" to "all of the time" for the HRQOL items. ${ }^{8}$ A higher SSS indicates worse symptoms, while a higher HRQOL subscale score indicates a better quality of life. Women with an SSS $<20$ in combination with an HRQOL score $>80$ were considered asymptomatic. ${ }^{9}$ If a patient had an additional UAE or a hysterectomy during follow-up, the timing was recorded.

The NRS for pain is a single 11-point numeric scale, with 0 representing one pain extreme (eg, "no pain") and 10 representing the other pain extreme (eg, "pain as bad as you can imagine" or "worst pain imaginable").
Postembolization symptoms and complications were registered and classified according to the quality improvement guidelines for percutaneous transcatheter embolization. $^{10}$

\section{Statistical Analysis}

Summary descriptive statistics were used for demographic parameters. SSS, HRQOL, JZ thickness, and uterine volume were compared in patients with pure $\mathrm{AD}$ and $\mathrm{AD}$ with fibroids. The JZ thickness and uterine volume were compared between the asymptomatic patients and the insufficient response patients in a three-month follow-up MRI. A p-value $<0.05$ was considered statistically significant. Statistical analysis was performed using SPSS 23.0 software for Windows (SPSS Inc., Chicago, Illinois, USA).

\section{Results}

\section{Technical Results}

Twenty-six (96\%) of the patients underwent bilateral UAE, and one underwent unilateral UAE due to the right uterine artery being absent. Only one patient with an ovarian artery supplying the AD had an OAE performed.

\section{Clinical Results}

Different scales of abdominal pain were observed in 25 of the patients $(92.6 \%)$, but these symptoms were relieved during the first week after the procedure, and eight patients presented with a foul vaginal discharge. One patient was suspected of having endometritis and needed broadspectrum antibiotics. Transient amenorrhea occurred in four women, but no major complications occurred.

The NRS pain score decreased from a median of 8 (5$10)$ at baseline to a median of $2(0-5)$ at the 42-month follow-up. There was a significant difference in the NRS before and after the treatment $(\mathrm{p}<0.05)$.

During a median clinical follow-up of 42 months (range 24-71), 26 of 27 patients had a preserved uterus, but one patient underwent a hysterectomy because the UAE had not improved their condition completely at 35 months. The total SSS decreased from a median of 59 (range 34-78) at baseline to a median of 9 (range 3-47) at 42 months; the HRQOL score increased from a median of 38 (range 23-49) at baseline to a median of 84 (range 46-97) at 42 months. The SSS and HRQOL scores for patients with pure adenomyosis and adenomyosis with fibroids are presented in Table 1. The SSS and the 
HRQOL scores show no statistical differences between the groups before and after UAE ( $\mathrm{p}=0.873,0.729,0.365$, and 0.710) (see Table 1).

Twenty out of the 27 women had SSS $<20$ combined with an overall HRQOL score $>80$, indicating they were asymptomatic. The clinical response of the remaining seven women was little improvement in their symptoms. One of these seven women underwent a hysterectomy at 35 months. There was a significant difference in the SSS and HRQOL scores between the asymptomatic group and the insufficient response group after UAE ( $p<0.001$ for all) (see Table 2).

\section{MRI Results}

Twenty-two patients had an MRI three months after the UAE. Of these 22 women, 14 women had pure AD, and eight women had $\mathrm{AD}$ combined with fibroids. The JZ thickness of these 22 patients at baseline was a median of $33 \mathrm{~mm}$ (range 15-61 mm) and at three months a median of $24 \mathrm{~mm}$ (range $12-40 \mathrm{~mm}$ ). There was no difference in JZ thickness between the patients with pure AD or AD combined with fibroids before and after UAE $(p=0.732$ and 0.973) (see Table 3).

The uterus volume of these 22 patients at baseline was a median of $304 \mathrm{~cm}^{3}$ (range $76-726 \mathrm{~cm}^{3}$ ) and at three months a median of $151 \mathrm{~cm}^{3}$ (range $58-346 \mathrm{~cm}^{3}$ ). Uterus volume reduction was $151 \mathrm{~cm}^{3}$ (48.3\%) (see Figure 1). There was no difference in uterus volume between the

Table I Clinical Follow-Up at Baseline and 42 Months After Uterine Artery Embolization in Women with Symptomatic Adenomyosis with or without Fibroids

\begin{tabular}{|l|l|l|l|}
\hline Parameters & $\begin{array}{l}\text { Women with } \\
\text { Adenomyosis } \\
(\mathbf{n}=19)\end{array}$ & $\begin{array}{l}\text { Women with } \\
\text { Adenomyosis and } \\
\text { Concurrent } \\
\text { Fibroids (n=8) }\end{array}$ & P value \\
\hline SSS & $59(34,78)$ & $55(38,69)$ & 0.873 \\
\hline Baseline & $9(3,47)$ & $12(4,38)$ & 0.729 \\
\hline $\begin{array}{l}\text { 42-month } \\
\text { follow-up }\end{array}$ & 47 & 45 & 0.790 \\
\hline Reduction & $48(23,49)$ & $32(23,47)$ & 0.365 \\
\hline HRQOL & $84(46,97)$ & $85(47-94)$ & 0.710 \\
\hline Baseline & 32 -month & & 0.873 \\
\hline follow-up & 48 & 50 & \\
\hline Increase & 45 &
\end{tabular}

Table 2 Clinical Follow-Up at Baseline and 42 Months After Uterine Artery Embolization Between Asymptomatic Women and Women with Insufficient Clinical Response

\begin{tabular}{|l|l|l|l|}
\hline Parameters & \multicolumn{1}{|l|}{$\begin{array}{l}\text { Asymptomatic } \\
\text { Women (n=20) }\end{array}$} & $\begin{array}{l}\text { Women with } \\
\text { Insufficient } \\
\text { Clinical } \\
\text { Response (n=7) }\end{array}$ & P value \\
\hline SSS & $59(34,75)$ & $53(38,78)$ & 0.541 \\
\hline Baseline & \multicolumn{5}{|l|}{$31(22,47)$} & 0.000 \\
\hline $\begin{array}{l}\text { 42-month } \\
\text { follow-up }\end{array}$ & $8(3,19)$ & 21 & 0.001 \\
\hline Reduction & 52 & $41(23,47)$ & 0.359 \\
\hline HRQOL & $34(23,49)$ & $49(46,68)$ & 0.000 \\
\hline Baseline & $89(80,97)$ & 16 & 0.000 \\
\hline $\begin{array}{l}42-m o n t h \\
\text { follow-up }\end{array}$ & 55 & & \\
\hline Increase & & & \\
\hline
\end{tabular}

patients with pure $\mathrm{AD}$ or $\mathrm{AD}$ combined with fibroids ( $\mathrm{p}$ $=0.838$ and $>0.99)($ see Table 3$)$.

Of these 22 women, 16 women were asymptomatic, and six women had an insufficient clinical response. There was no difference in JZ thickness and uterus volume between the subgroups after UAE ( $\mathrm{p}=0.912$ and 0.531$)$ (see Table 4).

Table 3 Magnetic Resonance Imaging Follow-Up at Baseline and Three Months After Uterine Artery Embolization in Women with Symptomatic Adenomyosis with or without Fibroids

\begin{tabular}{|c|c|c|c|}
\hline Parameters & $\begin{array}{l}\text { Women with } \\
\text { Adenomyosis } \\
(n=14)\end{array}$ & $\begin{array}{l}\text { Women with } \\
\text { Adenomyosis and } \\
\text { Concurrent } \\
\text { Fibroids }(n=8)\end{array}$ & $P$ value \\
\hline \multicolumn{4}{|c|}{ JZ thickness (mm) } \\
\hline Baseline & $39(15,61)$ & $32(26,49)$ & 0.732 \\
\hline $\begin{array}{l}\text { 3-month } \\
\text { follow-up }\end{array}$ & $26(12,40)$ & $23(16,34)$ & 0.973 \\
\hline Reduction & 12 & 11 & 0.373 \\
\hline \multicolumn{4}{|c|}{ Uterus volume $\left(\mathrm{cm}^{3}\right)$} \\
\hline Baseline & $325(76,727)$ & $236(148,611)$ & 0.838 \\
\hline $\begin{array}{l}\text { 3-month } \\
\text { follow-up }\end{array}$ & $156(58,346)$ & $147(76,235)$ & 1.000 \\
\hline Reduction & 146 & 110 & 0.633 \\
\hline
\end{tabular}


A

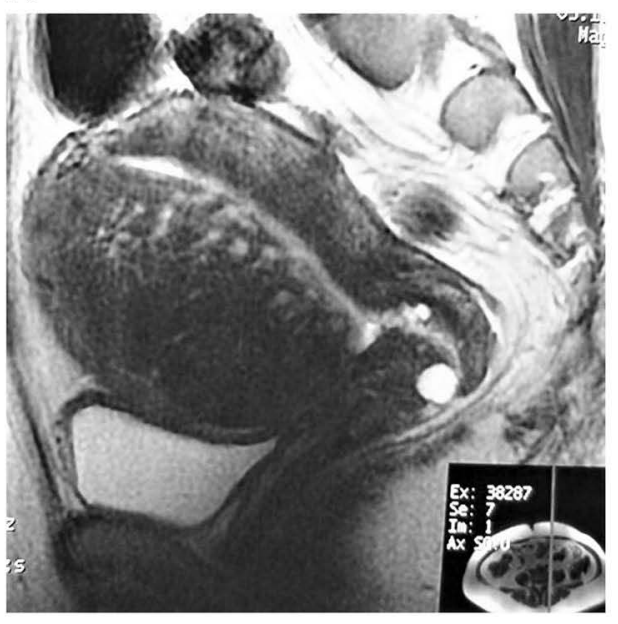

C
B

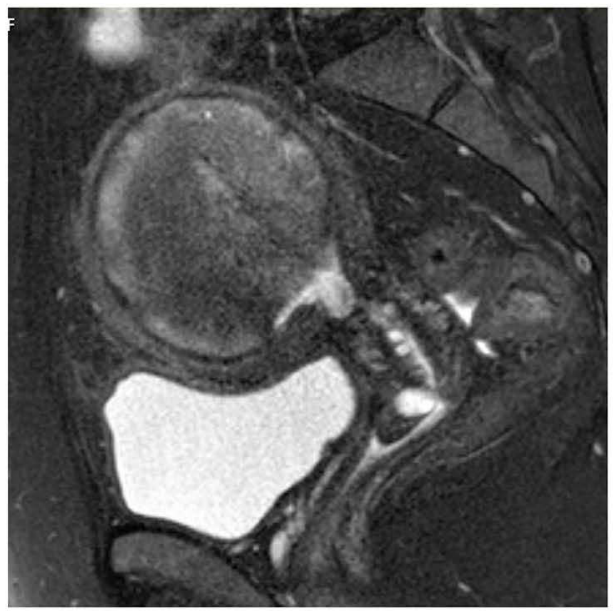

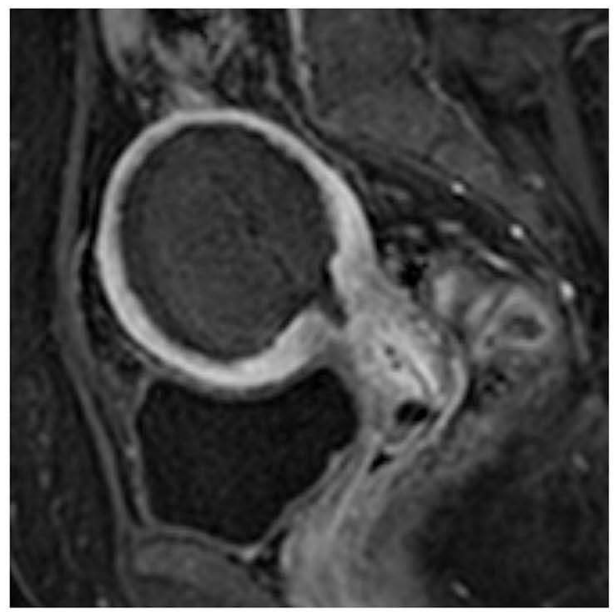

Figure I A 43-year-old woman who underwent uterine artery embolization for symptomatic adenomyosis. (A) Sagittal T2-weighted magnetic resonance imaging obtained before uterine artery embolization shows diffuse junctional zone thickening of the uterus, especially in the anterior wall. The volume of the uterus was $608 \mathrm{~cm}^{3}$. (B) Sagittal T2-weighted magnetic resonance imaging obtained three months after uterine artery embolization shows the decreased size of the adenomyosis and the uterus. The volume of the uterus was $212 \mathrm{~cm}^{3}$. (C) Sagittal contrast-enhanced TI-weighted magnetic resonance imaging obtained three months after uterine artery embolization shows that the adenomyosis was almost completely necrotic.

\section{Discussion}

In this study, the embolization began with smaller-sized PVA particles $(100 \mu \mathrm{m})$ to first block the smaller intra-AD vessels and then relatively larger particles $(300 \mu \mathrm{m})$ were used for the proximal embolization. Only one patient underwent a hysterectomy at 35 months. The preserved uterus rate was $96 \%(26 / 27)$, and the asymptomatic rate was $74 \%(20 / 27)$.

In previous studies, various embolization materials and protocols were used in UAE. However, it is not yet clear which embolization material or protocol can result in better clinical outcomes. Popovic et $\mathrm{al}^{7}$ summarized the results of 15 studies with a total of 511 cases. Improvements in symptoms that primarily related to bleeding, pain, and bulk-related discomfort were reported by 387 patients (75.7\%) at the 26.9 months follow-up. The embolization materials included PVA particles, TGM, KMG, and GFP, and the primary sizes were 355-500 $\mu \mathrm{m}, \quad 500-700 \mu \mathrm{m}$, and 700-900 $\mu \mathrm{m}$. However, the researchers did not perform a systematic literature search and performed only a limited qualitative assessment, so the symptom improvement rate should be interpreted critically.

De Bruijn et $\mathrm{al}^{9}$ also used the UFS-QoL to measure SS and HRQOL. Embolization was started with Embozene $500 \mu \mathrm{m}$ first to block the smaller vessels and continued with Embozene $700 \mu \mathrm{m}$, followed by Embozene $900 \mu \mathrm{m}$, when judged necessary. Five out of 28 women had 
Table 4 Magnetic Resonance Imaging Follow-Up at Baseline and Three Months After Uterine Artery Embolization Between Asymptomatic Women and Women with Insufficient Clinical Response

\begin{tabular}{|l|l|l|l|}
\hline Parameters & $\begin{array}{l}\text { Asymptomatic } \\
\text { Women (n=16) }\end{array}$ & $\begin{array}{l}\text { Women with } \\
\text { Insufficient } \\
\text { Clinical } \\
\text { Response (n=6) }\end{array}$ & P value \\
\hline JZ thickness (mm) & $32(15,61)$ & 0.854 \\
\hline Baseline & $35(17,49)$ & $22(12,40)$ & 0.912 \\
\hline $\begin{array}{l}\text { 3-month } \\
\text { follow-up }\end{array}$ & $24(13,34)$ & 10 & 0.318 \\
\hline Reduction & 12 & $171(122,727)$ & 0.253 \\
\hline Uterus volume (cm $\left.{ }^{3}\right)$ & $334(76,611)$ & $133(76,346)$ & 0.531 \\
\hline Baseline & $161(58,276)$ & 43 & 0.224 \\
\hline $\begin{array}{l}\text { 3-month } \\
\text { follow-up }\end{array}$ & 156 & & \\
\hline Reduction & &
\end{tabular}

undergone a secondary hysterectomy before the sevenyear follow-up, meaning a hysterectomy was avoided in $23(82 \%)$ cases, and $17(61 \%)$ of the 28 patients were asymptomatic. In a group of 40 patients studied by Smeets et $\mathrm{al}^{11}$ embospheres of 500-700 and 700-900 $\mu \mathrm{m}$ were used as embolic agents, and $83 \%$ of the patients had a preserved uterus and $72 \%$ of them were asymptomatic at a mean follow-up of 65 months.

The outcomes of this study are better than those of previous studies with medium-sized embolic particles (300-700 $\mu \mathrm{m})$, especially with respect to the preserved uteri. It is reasonable to assume that smaller-sized particles may induce larger ischemia with a more distal penetration into the lesions, leading to higher infarction and better clinical outcomes. ${ }^{12}$ Small-sized PVA particles have been reported as safe for UAE without untargeted embolization by Kim et al. ${ }^{13}$ In the study, UAE with the "1-2-3 protocol" (small PVA particles measuring $150-250 \mu \mathrm{m}$ were used at the beginning of embolization, followed by $250-355 \mu \mathrm{m}$ and $355-500 \mu \mathrm{m}$ particles) resulted in more than $80 \%$ of patients with complete necrosis of $\mathrm{AD}$ and a lower recurrence rate of symptoms. As in the Kim et $\mathrm{al}^{13}$ and de Bruijn et $\mathrm{al}^{9}$ studies, there were no deaths or other complications after UAE in this study.
This study found no difference in the reduction of SSS and an improvement in HRQOL between patients with pure $\mathrm{AD}$ and those with concomitant fibroids. This finding was concordant with the report of Smeets et al. ${ }^{11}$ In other words, the relief of symptoms and improvement of QoL after UAE was not related to whether the patients had pure $\mathrm{AD}$ or $\mathrm{AD}$ combined with uterine fibroids.

The study of de Bruijn et al $^{9}$ shows no HRQOL difference between patients with pure $\mathrm{AD}$ and those with $\mathrm{AD}$ and fibroids at the seven-year follow-up, but the SSS has a statistical difference in favor of those with AD combined with fibroids. Subsequently, de Bruijn et $\mathrm{al}^{14}$ published a meta-analysis suggesting that combined $\mathrm{AD}$ seems to respond better to $\mathrm{UAE}$ compared with pure $\mathrm{AD}$ in both the short-term and the long-term. Although several case series and cohorts that evaluated UAE as a treatment for symptomatic $\mathrm{AD}$ have been reported over the last fifteen years, many of the individual studies did not report all the information necessary to weigh up the quality of the research properly. Thus, there is still a lack of firm evidence that the outcome of UAE for AD combined with fibroids is better than that of pure $\mathrm{AD}$. Confirmation is imperative to studies in related randomized controlled trials. ${ }^{4,15-18}$

Whether JZ thickness at baseline can be used as a predictor of response is also controversial. De Bruijn's meta-analysis indicated that greater JZ thickness was associated with a decreased effectiveness in UAE for treating adenomyosis. ${ }^{14}$ However, a study by Froeling et $\mathrm{al}^{19}$ indicated that treatment failure was not associated with either JZ thickness or the pattern of uterine AD (focal or diffuse). The results of this study are consistent with those of Froeling et al, which do not support the use of JZ thickness at baseline as a predictor for long-term efficacy.

The biggest shortcoming of this retrospective study is that the sample size is too small. Secondly, T1-weighted contrast-enhanced images were absent on the three-month follow-up MRI in some patients, making it impossible to determine whether the percentage of necrosis of $A D$ was related to symptom recurrence in the long-term follow-up.

\section{Conclusions}

The follow-up outcomes of this study confirmed that UAE with small particles is safe and effective in treating $A D$, especially in terms of preserving the uterus. The findings also indicate that there is no difference in clinical improvement between women treated with UAE for pure AD and 
those treated for $\mathrm{AD}$ in combination with fibroids. In addition, JZ thickness at baseline does not seem to be a predictor for the long-term efficacy of UAE.

\section{Acknowledgments}

We would like to acknowledge the hard and dedicated work of all the staff that implemented the intervention and evaluation components of the study.

\section{Funding}

This study was funded by National Natural Science Foundation of China (81471769) and Clinical Research Support Fund of PLA General Hospital (2019XXJSYX04).

\section{Disclosure}

The authors declare that they have no competing interests.

\section{References}

1. Antero MF, Ayhan A, Segars J, Shih IM. Pathology and pathogenesis of adenomyosis. Semin Reprod Med. 2020;38(2-03):108-118. doi:10.1055/s-0040-1718922

2. Horton J, Sterrenburg M, Lane S, Maheshwari A, Li TC, Cheong Y. Reproductive, obstetric, and perinatal outcomes of women with adenomyosis and endometriosis: a systematic review and meta-analysis. Hum Reprod Update. 2019;25(5):592-632.

3. Upson K, Missmer SA. Epidemiology of Adenomyosis. Semin Reprod Med. 2020;38(2-03):89-107. doi:10.1055/s-0040-1718920

4. de Bruijn AM, Lohle PN, Huirne JA, de Vries J, Twisk M, Hehenkamp WJ; QUESTA-Trial Group. Uterine artery embolization versus hysterectomy in the treatment of symptomatic adenomyosis: protocol for the randomized QUESTA Trial. JMIR Res Protoc. 2018;7 (3):e47. doi:10.2196/resprot.8512

5. Dueholm M. Minimally invasive treatment of adenomyosis. Best Pract Res Clin Obstet Gynaecol. 2018;51:119-137. doi:10.1016/j. bpobgyn.2018.01.016

6. Dessouky R, Gamil SA, Nada MG, Mousa R, Libda Y. Management of uterine adenomyosis: current trends and uterine artery embolization as a potential alternative to hysterectomy. Insights Imaging. 2019;10 (1):48. doi:10.1186/s13244-019-0732-8

7. Popovic M, Puchner S, Berzaczy D, Lammer J, Bucek RA. Uterine artery embolization for the treatment of adenomyosis: a review. $J$ Vasc Interv Radiol. 2011;22(7):901-909. doi:10.1016/j.jvir.2011.03.013
8. Spies JB, Coyne K, Guaou Guaou N, Boyle D, Skyrnarz-Murphy K, Gonzalves SM. The UFS-QOL, a new disease-specific symptom and health-related quality of life questionnaire for leiomyomata.. Obstet Gynecol. 2002;99(2):290-300. doi:10.1016/s0029-7844(01)01702-1

9. de Bruijn AM, Smink M, Hehenkamp WJK, et al. Uterine artery embolization for symptomatic adenomyosis: 7-year clinical follow-up using UFS-Qol questionnaire. Cardiovasc Intervent Radiol. 2017;40 (9):1344-1350. doi:10.1007/s00270-017-1686-1

10. Angle JF, Siddiqi NH, Wallace MJ, et al.; Society of Interventional Radiology Standards of Practice Committee. Quality improvement guidelines for percutaneous transcatheter embolization: society of interventional radiology standards of practice committee. $J$ Vasc Interv Radiol. 2010;21(10):1479-1486. doi:10.1016/j. jvir.2010.06.014

11. Smeets AJ, Nijenhuis RJ, Boekkooi PF, Vervest HAM, van Rooij WJ, Lohle PNM. Long-term follow-up of uterine artery embolization for symptomatic adenomyosis. Cardiovasc Intervent Radiol. 2012;35 (4):815-819. doi:10.1007/s00270-011-0203-1

12. Bae SH, Kim MD, Kim GM, et al. Uterine artery embolization for adenomyosis: percentage of necrosis predicts midterm clinical recurrence. $J$ Vasc Interv Radiol. 2015;26(9):1290-1296. doi:10.1016/j.jvir.2015.04.026

13. Kim MD. Uterine artery embolization for leiomyomas and adenomyosis: a pictorial essay based on our experience from 1300 cases. Korean J Radiol. 2019;20(10):1462-1473. doi:10.3348/kjr.2019.0205

14. de Bruijn AM, Smink M, Lohle PNM, et al. Uterine artery embolization for the treatment of adenomyosis: a systematic review and meta-analysis. J Vasc Interv Radiol. 2017;28(12):1629-1642. doi:10.1016/j.jvir.2017.07.034

15. Kossaï M, Penault-Llorca F. Role of hormones in common benign uterine lesions: endometrial polyps, leiomyomas, and adenomyosis. Adv Exp Med Biol. 2020;1242:37-58.

16. Bourdon M, Santulli P, Jeljeli M, et al. Immunological changes associated with adenomyosis: a systematic review. Hum Reprod Update. 2021;27(1):108-129. doi:10.1093/humupd/dmaa038

17. Huo ZF, Chen CL, Liu P, et al. Analysis of related factors on effects of uterine artery embolization in the treatment of dysmenorrhea of adenomyosis and the construction and validation of prediction model. Zhonghua Fu Chan Ke Za Zhi. 2016;51(9):650-656. doi:10.3760/ cma.j.issn.0529-567X.2016.09.003

18. Taran FA, Stewart EA, Brucker S. Adenomyosis: epidemiology, risk factors, clinical phenotype and surgical and interventional alternatives to hysterectomy. Geburtshilfe Frauenheilkd. 2013;73 (9):924-931. doi:10.1055/s-0033-1350840

19. Froeling V, Scheurig-Muenkler C, Hamm B, Kroencke TJ. Uterine artery embolization to treat uterine adenomyosis with or without uterine leiomyomata: results of symptom control and health-related quality of life 40 months after treatment. Cardiovasc Intervent Radiol. 2012;35(3):523-529. doi:10.1007/s00270-011-0254-3
International Journal of General Medicine

\section{Publish your work in this journal}

The International Journal of General Medicine is an international, peer-reviewed open-access journal that focuses on general and internal medicine, pathogenesis, epidemiology, diagnosis, monitoring and treatment protocols. The journal is characterized by the rapid reporting of reviews, original research and clinical studies
Dovepress

across all disease areas. The manuscript management system is completely online and includes a very quick and fair peer-review system, which is all easy to use. Visit http://www.dovepress.com/ testimonials.php to read real quotes from published authors. 